\title{
Editorial
}

\section{Implementation Aspects and Testbeds for MIMO Systems}

\author{
Thomas Kaiser, ${ }^{1}$ André Bourdoux, ${ }^{2}$ Markus Rupp, ${ }^{3}$ and Ulrich Heute ${ }^{4}$ \\ ${ }^{1}$ Department of Communication Systems, Faculty of Engineering, University of Duisburg-Essen, 47048 Duisburg, Germany \\ ${ }^{2}$ IMEC vzw, DESICS Division, Kapeldreef 75, 3001 Leuven, Belgium \\ ${ }^{3}$ Institute of Communications and RF Engineering, TU Wien, Gusshausstrasse 25/389, 1040 Wien, Austria \\ ${ }^{4}$ Institute for Circuits and Systems Theory, Faculty of Engineering, Christian-Albrechts-University Kiel, Kaiserstraße 2, \\ 24143 Kiel-Gaarden, Germany
}

Received 21 September 2005; Accepted 21 September 2005

Copyright (C) 2006 Thomas Kaiser et al. This is an open access article distributed under the Creative Commons Attribution License, which permits unrestricted use, distribution, and reproduction in any medium, provided the original work is properly cited.

\begin{abstract}
The MIMO (multiple-input multiple-output) systems have emerged as a key technology for wireless local area networks (WLANs), wireless metropolitan area networks (WMANs), and cellular mobile communication systems $(3 \mathrm{G}, 4 \mathrm{G})$ because they promise greater coverage, higher data rates, and improved link robustness by adding a spatial dimension to the time, the frequency, and the code dimensions. Recent progress in standardization and in first MIMO prototype chipsets has forced manufacturers worldwide to pay more attention to MIMO implementation aspects. Moreover, MIMO testbeds have become more and more attractive to universities and to research institutes as has been observed in the past few years. The aim of this special issue is to reflect the current state-of-the-art MIMO testbeds and to examine the several MIMO implementation challenges for current and for future wireless communication standards.

We classified the accepted thirteen submissions into four major categories: (1) hardware-oriented prototypes, (2) flexible testbeds, (3) analog issues, and (4) fast algorithms.
\end{abstract}

\section{Hardware-oriented prototypes}

In the first paper, Guo et al. present an efficient circulant approximation-based MIMO equalizer architecture for the CDMA downlink, reducing the direct matrix inverse (DMI) to some FFT operations. Further parallel and pipelined VLSI architectures with Hermitian optimization and reducedstate FFT reduce the complexity even more. A comparative study of both the conjugate-gradient and the DMI algorithms shows very promising performance/complexity tradeoff. VLSI design space in terms of area/time efficiency is explored extensively for layered parallelism and pipelining with a Catapult $\mathrm{C}$ high-level synthesis methodology. In the next paper, Dowle et al. describe the development of the STAR (space-time array research) platform, an FPGA-based research unit operating at $2.45 \mathrm{GHz}$ and capable of having a maximum of twelve $20 \mathrm{MHz}$ bandwidth channels of realtime, space-time, and MIMO processing. The design method starts with Matlab/Octave. With manual refinement steps, VHDL code for FPGAs is obtained and verified via ModelSim with the original design. Various pitfalls associated with the implementation of MIMO algorithms in real time are highlighted, and finally the development requirements are given to aid comparison with traditional DSP development. The paper by Goud et al. describes a portable $4 \times 4$ MIMO testbed operating in an ISM band around $900 \mathrm{MHz}$. Details of channel measurements and capacity analysis of unusual indoor and outdoor locations obtained with the test-bed are also included. The next paper by Haustein et al. presents a reconfigurable hardware test-bed suitable for real-time mobile communication with multiple antennas. Supported are four transmit and five receive antennas operating at $5.2 \mathrm{GHz}$ with a maximum bandwidth of $100 \mathrm{MHz}$. Efficient implementation of MIMO signal processing using FPGAs and DSPs is described. An experimental verification of several real-time MIMO transmission schemes at high data rates in a typical office scenario is presented, and results on the achieved BER and throughput performance are given. Spectral efficiencies of more than $20 \mathrm{bps} / \mathrm{Hz}$ and a throughput of more than $150 \mathrm{Mbps}$ was shown with a single-carrier transmission. The experimental results clearly show the feasibility of real-time high-data-rate MIMO techniques with state-of-the-art hardware and that more sophisticated baseband signal processing will be an essential part of future communication systems. 
Weijers et al. propose a systematic way from a transmissionsystem model, as often underlying a Matlab simulation, to a real-time prototype realized on a predefined hardware platform, avoiding inconsistencies of adhoc procedures. The suggested design flow is partly manual, but always systematic and assisted by tools suitable for the individual steps.

\section{Flexible testbeds}

The next five papers of the issue cover flexible testbeds, where the flexibility is usually achieved by higher-level programming languages. Xiang et al. describe a $4 \times 4$ MIMOOFDM test-bed mainly based on laboratory test equipment and offline processing. Channel measurements and antenna selection techniques are presented. The paper also assesses the degradation due to carrier frequency offset and imperfect channel estimation. The next paper by Borkowski et al. presents a real-time MIMO test-bed for both single-carrier and OFDM transmission. A specific SIMD processor implemented on FPGAs is described, as well as the specific analog hardware at $10 \mathrm{GHz}$ that is supported by offline and online calibration. The influence of polarization on the channel capacity is also addressed. In the paper by Caban et al., the focus is on the comfortable use of a flexible DSP/FPGA and RF hardware setup. Real-time tests with four transmit and receive channels each are possible at a data rate of $2.45 \mathrm{GHz}$. All pre- and postprocessing is done within Matlab, while the real-time requirements are fulfilled by burst-data transmission through the hardware. Multiuser abilities are also provided. In the contribution by Samuelsson et al., a test-bed for spatial multiplexing is proposed that relies on off-the-shelf radio hardware only. A comparison of SISO with MIMO reveals that even with rather low-cost hardware the remarkable spectral efficiency improvement and the associated multiplexing gain of MIMO can be demonstrated. The paper by Fàbregas et al. presents the complete design methodology of a MIMO-OFDM test-bed for WLAN applications. The design steps include a characterization of the indoor MIMO channel and the specific baseband and RF hardware at $5 \mathrm{GHz}$. The mapping and validation of the algorithms using Matlab, $\mathrm{C}++$, and VHDL is detailed, and measurements are described.

\section{Analog issues}

The contribution by Liu et al. addresses a specific problem in the popular transmit-antenna diversity scheme termed "transmit MRC." While symmetries are usually assumed for the up- and downlink channels as well as between the antennas, in reality mismatches are found. A novel statistical analysis provides a deeper understanding and especially leads to a novel calibration scheme, which is finally implemented on a real-time prototyping platform. The paper by Piechocki et al. presents an extension of analogue turbo decoder concepts to MIMO detection. The first analogue implementation results show reductions of a few orders of magnitude in the number of required transistors, consumed energy, and the same order of improvement in processing speed. LDPC is used as a test case for the analysis.

\section{Fast algorithms}

Safar et al. propose an efficient detection of space-frequency block codes by means of the sphere decoding technique formulated in the complex domain. Three approaches are detailed: one approach is modulation independent, whereas the two others are specific for QAM and QPSK, respectively. The complexity analysis of these techniques is assessed.

\section{ACKNOWLEDGMENTS}

We thank the authors, the reviewers, the publisher, and the Editor-in-Chief for their efforts. We also hope that this special issue will stimulate subsequent contributions on MIMO testbeds so as to widely spread the required technical knowledge and to validate in further detail the realistic performance gain of multiantenna systems.

\section{Thomas Kaiser André Bourdoux Markus Rupp Ulrich Heute}

Thomas Kaiser received a Diploma degree from the Ruhr-University Bochum in 1991, and a Ph.D. degree in 1995 and a German Habilitation degree in 2000, both from Gerhard-Mercator-University Duisburg and in electrical engineering. From 1995 to 1996, he spent a research leave at the University of Southern California, Los Angeles, which was grant-aided by the German Academic Exchange Service. From

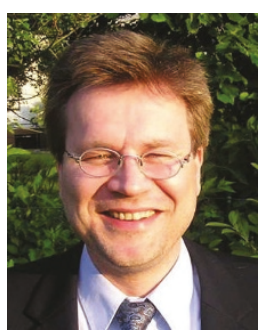
April 2000 to March 2001, he was the Head of the Department of Communication Systems at Gerhard-Mercator-University Duisburg and from April 2001 to March 2002, he was the Head of the Department of Wireless Chips \& Systems (WCS) at Fraunhofer Institute of Microelectronic Circuits and Systems. Now he is the Coleader of the Smart Antenna Research Team (SmART) at the University of Duisburg-Essen. In summer 2005, he joined Stanford's Smart Antenna Research Group (SARG) as a Visiting Professor. He has published more than 80 papers in international journals and at conferences, and he is the coeditor of the three forthcoming books: UWB Communication Systems - A Comprehensive Overview, Smart Antennas-State of the Art (both to appear in the EURASIP book series), and UWB Communications (to be published by $\mathrm{Wi}$ ley). He is the founder of PLANET MIMO Ltd. and belongs to the Editorial Board of EURASIP Journal of Applied Signal Processing and to the advisory board of a European multiantenna project. He is the founding Editor-in-Chief of the upcoming IEEE Signal Processing Society e-letter. He is involved in several national and international projects, and has chaired and cochaired a number of special sessions on multiantenna implementation issues. Beside this special issue in hand, he is also a Guest Editor of the EURASIP special issues on "Advances in Smart Antennas," "UWB State of the Art," and "Wireless Location Technologies and Applications." His current research interest focuses on applied signal processing with emphasis on multiantenna systems, especially its applicability to ultra-wideband systems, and on implementation issues. 
André Bourdoux received the M.S. degree in electrical engineering (specialization in microelectronics) in 1982 from the Université Catholique de Louvain-la-Neuve, Belgium. He is coordinating the research on multiantenna communications in the Wireless Research Group at IMEC. His current interests span the areas of wireless communications theory, signal processing, and transceiver architectures with a special em-

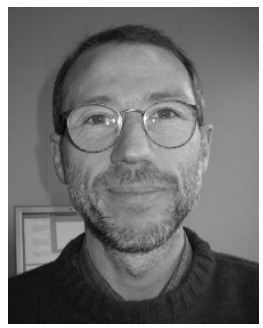
phasis on broadband and multiantenna systems. Before joining IMEC, his research activities were in the field of algorithms and RF architectures for coherent and high-resolution radar systems. $\mathrm{He}$ is the author and coauthor of several conference and journal papers and of 2 patents applications in the field of SDMA and MIMO transmission. He is a coeditor of the book Smart Antennas-State of the Art to be published in the EURASIP Book Series on Signal Processing and Communications.

Markus Rupp received his Dipl.-Ing. degree in 1988 from the University of Saarbruecken, Germany, and his Dr.-Ing. degree in 1993 from the Technische Universität Darmstadt, Germany. He is presently a Full Professor of digital signal processing in mobile communications at the Technical University of Vienna. He is an Associate Editor of IEEE Transactions on Signal Processing, of JASP EURASIP Journal of Applied

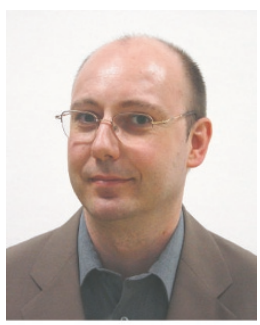
Signal Processing, of JES EURASIP Journal on Embedded Systems, and is elected AdCom Member of EURASIP. He authored and coauthored more than 180 papers and patents on adaptive filtering, wireless communications, and rapid prototyping.

Ulrich Heute was born in 1944 in Magdeburg, went to school till 1964, and studied electrical engineering at Stuttgart Technical University from 1965 to 1970 ; he received the Dipl.-Ing. degree in 1970. At Friedrich-Alexander University, Erlangen, he did research on digital filters, spectral analysis, and speech processing; he received the Ph.D. degree in 1975 and the Habilitation degree in 1982, and was a Senior Engi-

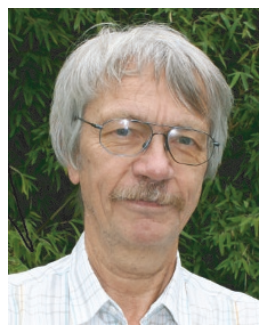
neer until 1987. He became a Professor for digital signal processing at Ruhr-University Bochum in 1987, and has been a Professor for circuit and system theory at Christian-Albrechts University, Kiel, since 1993. He was a Guest Researcher at Georgia Institute of Technology in 1979 and at the University of California/Santa Barbara in 1990, 1991, and 1997 (some months each). His research interests include digital signal processing, filters and filter banks, spectral analysis, and speech-signal processing (combined source and channel coding, enhancement, modeling, and quality assessment). He is a Member of the IEEE (SM), EURASIP (Secretary 1988-1994, President 1994-1998), ITG, DEGA, and ASA. 